\title{
Fetal Biparietal Diameter as a Potential Risk Factor for Emergency Cesarean Section due to Labor Arrest
}

\author{
Satoshi Shinohara, ${ }^{1}$ Atsuhito Amemiya ${ }^{1}$ and Motoi Takizawa ${ }^{1}$ \\ ${ }^{1}$ Department of Obstetrics and Gynecology, National Hospital Organization Kofu National Hospital, Kofu, \\ Yamanashi, Japan
}

\begin{abstract}
Labor arrest is the most common indication for emergency cesarean section (ECS). Increased biparietal diameter (BPD) has been reported as a risk factor for ECS due to labor arrest in different countries, but it is unclear whether this relationship is relevant in Japan. Considering the difference in maternal physique according to race and ethnicity, we retrospectively evaluated the association between ECS due to labor arrest and BPD, measured by ultrasonography $<7$ days before term deliveries in Japanese women. BPD is routinely measured in Japan for estimating fetal weight. Information was extracted from obstetric records at the National Hospital Organization Kofu National Hospital between January 2012 and November 2019. Patients with multiple pregnancies, instrumental (forceps or vacuum) delivery, elective cesarean sections, and ECS due to reasons other than labor arrest were excluded. Thus, 2,695 women were included (age, $31.3 \pm 6.2$ years; pre-pregnancy body mass index, $\left.20.9 \pm 3.2 \mathrm{~kg} / \mathrm{m}^{2}\right)$, and $1,319(48.9 \%)$ were nulliparous. The incidence of ECS due to labor arrest was $2.4 \%(64 / 2,695)$. Multivariable analysis indicated that BPD was significantly associated with ECS due to labor arrest (adjusted odds ratio, 1.12; 95\% confidence interval, 1.04-1.20). The optimal BPD cut-off value for predicting ECS due to labor arrest was $94 \mathrm{~mm}$ (area under the curve, 0.61; sensitivity, 53.1\%; specificity, 65.1\%). Despite the significant association with ECS, BPD is not useful to predict ECS due to labor arrest. Thus, mothers should be encouraged for attempting vaginal deliveries, even in the case of having babies with large fetal BPD.
\end{abstract}

Keywords: biparietal diameter; emergency cesarean section; labor arrest; prediction; ultrasound Tohoku J. Exp. Med., 2020 March, 250 (3), 161-166.

\section{Introduction}

Labor arrest is a condition that varies from slowerthan-normal progression or complete absence of labor caused by poor cervical ripening despite adequate myometrial contractions (Feinstein et al. 2002; Gifford et al. 2000; Bardin et al. 2016). As labor arrest is the most common indication for emergency cesarean section (ECS), which is associated with increased rates of maternal and fetal morbidities (Yang and Sun 2017), there has been much debate on the relationship between labor arrest and ECS (Gifford et al. 2000; Bardin et al. 2016; Karaaslan et al. 2019). However, predicting the risk of ECS due to labor arrest in the clinical setting is difficult. Therefore, a complete understanding of risk factors associated with labor arrest and the development of appropriate treatment strategies are essential. Labor arrest may be attributed to several factors, such as maternal physique, parity, hydramnios, fetal sex, premature rupture of membranes, labor induction, macrosomia, gestational diabetes mellitus (GDM), and hypertensive disorders of pregnancy (HDP) (Feinstein et al. 2002; Kominiarek et al. 2011; Toh-Adam et al. 2012; Lee et al. 2012). Antepartum sonographic fetal head biometry parameters, such as the head circumference (HC) (Lipschuetz et al. 2018) and biparietal diameter (BPD) (Bardin et al. 2016; Karaaslan et al. 2019), have been used to assess the risk of labor arrest in recent years; previous studies have reported that increased HC and BPD are independent factors that increase risk of ECS due to labor arrest (Bardin et al. 2016; Lipschuetz et al. 2018; Karaaslan et al. 2019). However, to the best of our knowledge, no studies have assessed the relationship between antepartum sonographic fetal head biometry parameters and ECS due to labor arrest in the Japanese population. Furthermore, it remains unclear

Received December 17, 2019; revised and accepted February 20, 2020. Published online March 6, 2020; doi: 10.1620/tjem.250.161. Correspondence: Satoshi Shinohara, Department of Obstetrics and Gynecology, National Hospital Organization Kofu National Hospital, 11-35 Tenjin, Kofu, Yamanashi 400-8533, Japan.

e-mail: shinohara617@gmail.com

(C)2020 Tohoku University Medical Press. This is an open-access article distributed under the terms of the Creative Commons Attribution-NonCommercial-NoDerivatives 4.0 International License (CC-BY-NC-ND 4.0). Anyone may download, reuse, copy, reprint, or distribute the article without modifications or adaptations for non-profit purposes if they cite the original authors and source properly.

https://creativecommons.org/licenses/by-nc-nd/4.0/ 
whether this relationship is relevant to the Japanese population, considering that maternal physique differs according to race and ethnicity (Deurenberg et al. 2002). In particular, pregnant women in Japan are physically shorter and thinner than in Western countries (Deurenberg et al. 2002). Prenatal check-ups in Japan are conducted once weekly from 36 to 39 weeks and twice weekly after 40 weeks (Minakami et al. 2014). Moreover, Japanese obstetricians calculate the BPD at each prenatal check-up to obtain the estimated fetal weight (Minakami et al. 2014). Consequently, in term pregnancies, sonographic measurements of BPD within 7 days prior to delivery are readily available to obstetricians in the clinical setting. Improved understanding of the relationship between BPD before delivery and ECS due to labor arrest will provide clinically useful information for perinatal management in Japan. We conducted this retrospective study to evaluate the association between fetal BPD, measured by ultrasonography prior to delivery in term pregnancies, and ECS due to labor arrest in a Japanese cohort.

\section{Materials and Methods}

\section{Study design}

We conducted a retrospective observational cohort study at the National Hospital Organization Kofu National Hospital (a community hospital) between January 2012 and November 2019. The study included women with singleton pregnancies at $37+0$ to $41+6$ weeks' gestation, whose sonographic measurements of BPD within 7 days before delivery were available. Based on the methodology of previous studies (Bardin et al. 2016; Lipschuetz et al. 2018; Karaaslan et al. 2019), we excluded women with multiple pregnancies $(n=24)$, those with instrumental (forceps or vacuum) delivery $(\mathrm{n}=94)$, those with missing data $(\mathrm{n}=$ 11), those who underwent an elective cesarean section ( $\mathrm{n}=$ 423), and those who underwent an ECS for indications other than labor arrest $(n=80)$. Indications for elective cesarean sections included a history of previous cesarean section, breech presentation, placenta previa or low-lying placenta, prior myomectomy, and maternal disorders. Indications for ECS included labor arrest, non-reassuring fetal status, severe hypertension (BP $\geq 160 / 110 \mathrm{mmHg}$ and uncontrolled by medication), placenta abruption, HELLP syndrome (Hemolysis, Elevated Liver enzyme, and Low platelet), and others. The study protocol was reviewed and approved by the Human Subjects Review Committee of the National Hospital Organization Kofu National Hospital, and the requirement for acquisition of informed consent from patients was waived owing to the retrospective study design. Nevertheless, patients were provided with the opportunity to refuse the usage of their data through the hospital's website. All procedures were performed in accordance with the 1964 Helsinki Declaration and its later amendments.

\section{Data collection}

We collected obstetric data from medical and operative records. Gestational age was determined on the basis of the maternally reported last menstrual period and was confirmed by the crown-rump length measured on the first-trimester sonogram. We recorded data on the mother's age at delivery, use of in vitro fertilization, parity, gestational age at delivery, maternal stature, and pre-pregnancy weight status. Additionally, we assessed fetal sex and the presence of GDM, HDP, and macrosomia, which are potential confounding factors that have been reported to be risk factors for labor arrest (Feinstein et al. 2002; Kominiarek et al. 2011; Toh-Adam et al. 2012; Lee et al. 2012). The dose and type of uterine contraction agent (oxytocin or prostaglandin) administered were determined by the treating obstetrician during labor induction or augmentation, according to the guidelines for obstetrical practice in Japan (Minakami et al. 2014). Our facility does not perform mechanical labor induction such as the use of a balloon to stretch the cervix. The fetal BPD was measured from the outer edge of the proximal calvaria to the inner edge of the distal calvarial wall (outer-inner) at the level of the third ventricle and thalami (Galan et al. 2008; Bardin et al. 2016). To identify potential indicators of ECS, we defined labor arrest as follows: arrested labor for $>2 \mathrm{~h}$ in either the first or second stage of labor (Minakami et al. 2014; Nishimura et al. 2016). A diagnosis of GDM was made if there was at least one abnormal plasma glucose value $(\geq 92$, 180 , and $153 \mathrm{mg} / \mathrm{dL}$ among fasting, 1-h, and 2-h plasma glucose concentrations, respectively) after a 75 -g oral glucose tolerance test (Minakami et al. 2014). HDP was defined as a blood pressure level $\geq 140 / 90 \mathrm{mmHg}$ on at least two occasions (Ohkuchi et al. 2017). In a previous study, short stature was defined by a cut-off value at the $5^{\text {th }}$ percentile ranking (Toh-Adam et al. 2012) and was consequently defined as height $<150 \mathrm{~cm}$ in this study. Body mass index (BMI) before pregnancy was calculated based on World Health Organization standards, and patients were classified as obese $\left(\geq 25.0 \mathrm{~kg} / \mathrm{m}^{2}\right)$ or non-obese $(<25.0 \mathrm{~kg} /$ $\mathrm{m}^{2}$ ) according to the Japan Society of Obstetrics and Gynecology Guidelines for Obstetrical Practice 2014 (Minakami et al. 2014). Macrosomia was defined as birth weight $>4,000 \mathrm{~g}$ (Boulet et al. 2003).

\section{Statistical analyses}

The Mann-Whitney U and Chi-squared tests were first used to determine the potential confounding factors for ECS due to labor arrest. Next, a multiple logistic regression model was used to identify variables significantly associated with ECS due to labor arrest. A receiver-operating characteristic (ROC) curve was subsequently used to determine the optimal BPD cut-off value for predicting ECS due to labor arrest. We used the Youden index, which describes the maximum vertical distance between the ROC curve and the diagonal or chance line, to define the optimal cut-off value (Perkins and Schisterman 2006). All analyses were 
performed using Bell Curve for Excel (Social Survey Research Information Co., Ltd., Tokyo, Japan), and the significance level was set at $\mathrm{P}<0.05$.

\section{Results}

A total of 2,695 women were considered eligible for inclusion in this study. The mean maternal age was $31.3 \pm$ 6.2 years, and the mean maternal pre-pregnancy BMI was $20.9 \pm 3.2 \mathrm{~kg} / \mathrm{m}^{2}$, with 1,319 nulliparous women (48.9\%), 1,314 male infants (48.8\%), 163 women with GDM (6.0\%), 82 women with HDP (3.0\%), and 32 macrosomic infants $(1.2 \%)$. Table 1 summarizes the clinical characteristics of women enrolled in this study. The overall incidence rate of ECS due to labor arrest was $2.4 \%(64 / 2,695)$. Maternal age, nulliparity, gestational age, pre-pregnancy BMI, pretreatment with uterine contraction agent, male infant and birth weight were significantly higher in patients who underwent ECS due to labor arrest than in those who had normal vaginal delivery, whereas maternal height was significantly lower (Table 1).

There was a statistically significant difference in ECS due to labor arrest among the quartile groups (Table 2). As demonstrated in Table 2, there were significant differences in ECS due to labor arrest among the four groups of women, and the higher the BPD value, the higher the number of ECSs performed due to labor arrest. In multivariable analyses, the adjusted odds ratio (aOR) for ECS due to labor arrest was 1.12 (95\% confidence interval [CI], 1.04 to $1.20 ; \mathrm{P}=0.003$ ) for each $1-\mathrm{mm}$ increase in BPD.
Moreover, short stature (aOR, 3.07; 95\% CI, 1.23-7.64), HDP (aOR, 6.10; 95\% CI, 2.81-13.2), nulliparity (aOR, 13.2; 95\% CI, 5.18-33.5), GDM (aOR, 3.02; 95\% CI, 1.366.69 ), and macrosomia (adjusted OR, 6.19; 95\% CI, 1.5025.4) were found to be associated with ECS due to labor arrest (Table 3).

The ROC curve analysis suggested that a BPD cut-off value of $94 \mathrm{~mm}$ would allow for a maximum number of patients to be correctly classified according to ECS due to labor arrest. A cut-off point of $94 \mathrm{~mm}$ yielded a sensitivity of $53.1 \%$, specificity of $65.1 \%$, positive predictive value of $3.6 \%$, and negative predictive value of $98.2 \%$ (area under the curve: 0.61) (Fig. 1).

We also conducted a similar analysis by extracting 1,906 women with normal physique $(18.5<\mathrm{BMI}<25.0 \mathrm{~kg} /$ $\mathrm{m}^{2}$ ) (Minakami et al. 2014), because they comprised the majority of this study population. The overall incidence rate of ECS due to labor arrest was 2.6\% $(49 / 1,906)$. BPD was significantly different in patients who underwent ECS due to labor arrest than in those who had normal vaginal delivery $(93.4 \pm 43.8$ vs. $92.0 \pm 3.6 \mathrm{~mm}, \mathrm{P}=0.02)$. Based on ROC analysis for 1,906 women with normal physique, a BPD cut-off value of $94 \mathrm{~mm}$ would allow for the maximum number of women to be correctly classified according to ECS due to labor arrest. A cut-off value of $94 \mathrm{~mm}$ provided a sensitivity of $51.0 \%$, specificity of $65.8 \%$, positive predictive value of $3.8 \%$, and negative predictive value of $98.1 \%$ (area under the curve: 0.59) (Fig. 2).

Table 1. Baseline characteristics of the study population.

\begin{tabular}{lccr}
\hline & $\begin{array}{c}\text { Normal vaginal delivery } \\
(\mathrm{n}=2,631)\end{array}$ & $\begin{array}{c}\text { ECS due to labor arrest } \\
(\mathrm{n}=64)\end{array}$ & P value \\
\hline Maternal age, years & $31.3 \pm 6.2$ & $32.5 \pm 6.3$ & 0.03 \\
Nulliparity & $1,260(47.9)$ & $59(92.2)$ & $<0.001$ \\
Gestational age, weeks & $39.3 \pm 1.1$ & $40.0 \pm 1.0$ & $<0.001$ \\
Maternal height & $158.4 \pm 5.4$ & $156.1 \pm 5.6$ & $<0.001$ \\
Pre-pregnancy BMI, $\mathrm{kg} / \mathrm{m}^{2}$ & $20.9 \pm 3.2$ & $21.9 \pm 3.8$ & 0.005 \\
IVF & $83(3.2)$ & $3(4.7)$ & 0.56 \\
Birth weight, g & $3,077 \pm 366.1$ & $3,303 \pm 389.6$ & $<0.001$ \\
Pretreatment with a uterine contraction agent & $786(29.9)$ & $52(81.3)$ & $<0.001$ \\
Male infant & $1,275(48.4)$ & $39(60.9)$ & 0.048 \\
\hline
\end{tabular}

Values are presented as an average \pm standard deviation or as numbers $(\%)$.

ECS, emergency cesarean section; BMI, body mass index; IVF, in vitro fertilization.

Table 2. Prevalence of emergency cesarean section due to labor arrest according to fetal biparietal diameter quartiles measured within 7 days prior to delivery.

\begin{tabular}{lccccc}
\hline & $\begin{array}{c}\text { Group 1 } \\
\text { (BPD 71-89 mm) } \\
\mathrm{n}=676\end{array}$ & $\begin{array}{c}\text { Group 2 } \\
\text { (BPD 90-92 mm) } \\
\mathrm{n}=786\end{array}$ & $\begin{array}{c}\text { Group 3 } \\
\text { (BPD 93-95 mm) } \\
\mathrm{n}=733\end{array}$ & $\begin{array}{c}\text { Group 4 } \\
\text { (BPD 96-104 mm) } \\
\mathrm{n}=500\end{array}$ & P value \\
\hline ECS due to labor arrest & $9(1.3)$ & $17(2.2)$ & $18(2.5)$ & $20(4.0)$ & 0.03 \\
\hline
\end{tabular}

Values are presented as numbers (\%).

ECS, emergency cesarean section; BPD, biparietal diameter. 
Table 3. Factors associated with emergency cesarean section due to labor arrest.

\begin{tabular}{|c|c|c|c|c|c|c|}
\hline \multirow{2}{*}{ Variables } & \multirow{2}{*}{$\begin{array}{l}\text { ECS due to labor arrest } \\
\text { (n) }\end{array}$} & \multirow{2}{*}{$\begin{array}{l}\text { Normal vaginal delivery } \\
\text { (n) }\end{array}$} & \multicolumn{2}{|c|}{ Crude } & \multicolumn{2}{|c|}{ Adjusted } \\
\hline & & & OR & $95 \% \mathrm{CI}$ & OR & $95 \% \mathrm{CI}$ \\
\hline $\begin{array}{c}\text { BPD in mm } \\
\text { (median }\left[25^{\text {th }}-75^{\text {th }} \text { percentile }\right] \text { ) }\end{array}$ & $94.0(91.0-96.0)$ & $92.0(89.0-95.0)$ & & & 1.12 & $1.04-1.20$ \\
\hline \multicolumn{7}{|l|}{ Maternal stature } \\
\hline$\geq 150 \mathrm{~cm}$ & 58 & 2,521 & 1.0 & Reference & 1.0 & Reference \\
\hline$<150 \mathrm{~cm}$ & 6 & 110 & 2.37 & $1.001-5.61$ & 3.07 & $1.23-7.64$ \\
\hline \multicolumn{7}{|l|}{ Pre-pregnancy BMI } \\
\hline$<25.0 \mathrm{~kg} / \mathrm{m}^{2}$ & 57 & 2,392 & 1.0 & Reference & 1.0 & Reference \\
\hline$\geq 25.0 \mathrm{~kg} / \mathrm{m}^{2}$ & 7 & 239 & 1.22 & $0.55-2.72$ & 1.36 & $0.57-3.22$ \\
\hline \multicolumn{7}{|l|}{ Nulliparity } \\
\hline No & 5 & 1,371 & 1.0 & Reference & 1.0 & Reference \\
\hline Yes & 59 & 1,260 & 12.8 & $5.14-32.9$ & 13.2 & $5.18-33.5$ \\
\hline \multicolumn{7}{|l|}{ GDM } \\
\hline No & 55 & 2,477 & 1.0 & Reference & 1.0 & Reference \\
\hline Yes & 9 & 154 & 2.63 & $1.28-5.43$ & 3.02 & $1.36-6.69$ \\
\hline \multicolumn{7}{|l|}{ HDP } \\
\hline No & 54 & 2,559 & 1.0 & Reference & 1.0 & Reference \\
\hline Yes & 10 & 72 & 6.58 & $3.22-13.4$ & 6.10 & $2.81-13.2$ \\
\hline \multicolumn{7}{|l|}{ Birth weight } \\
\hline$<4,000 \mathrm{~g}$ & 61 & 2,602 & 1.0 & Reference & 1.0 & Reference \\
\hline$\geq 4,000 \mathrm{~g}$ & 3 & 29 & 4.41 & $1.31-14.9$ & 6.19 & $1.50-25.5$ \\
\hline
\end{tabular}

ECS, emergency cesarean section; BPD, biparietal diameter; BMI, body mass index; GDM, gestational diabetes mellitus; HDP, hypertensive disorders of pregnancy.

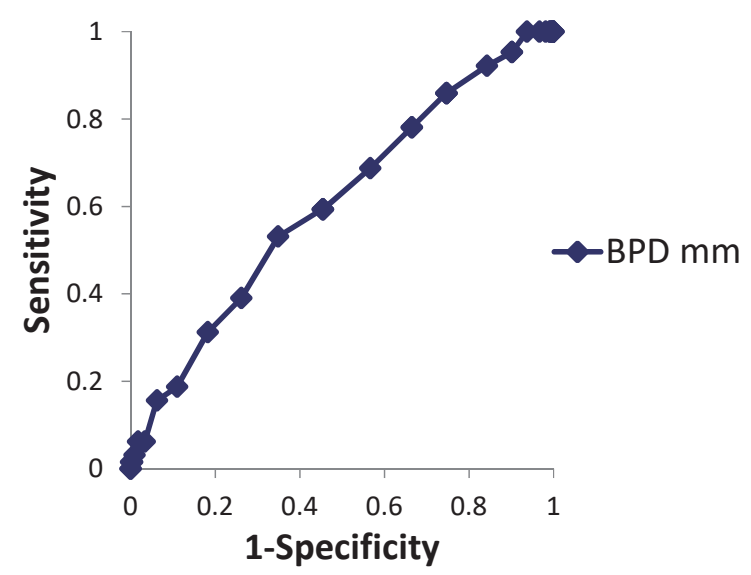

Fig. 1. Receiver-operating curve analysis of BPD for predicting ECS in a cohort of 2,695 women.

Receiver-operating curve analysis was performed for determining the optimal BPD cut-off value for predicting ECS due to labor arrest in a cohort of 2,695 women at 37-41 weeks' gestation.

ECS, emergency cesarean section; BPD, biparietal diameter.

\section{Discussion}

In this study on Japanese women, two important clinical findings were obtained. (1) Increased BPD was found

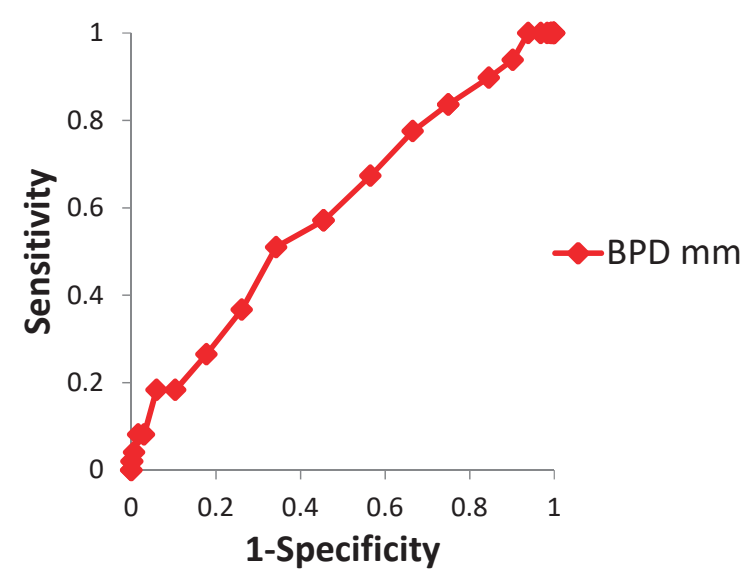

Fig. 2. Receiver-operating curve analysis of BPD for predicting ECS in a cohort of 1,906 women with normal physique. Receiver-operating curve analysis was performed to determine the optimal BPD cut-off value for predicting ECS due to labor arrest in a cohort of 1,906 women with normal physique $\left(18.5<\mathrm{BMI}<25.0 \mathrm{~kg} / \mathrm{m}^{2}\right)$ at $37-41$ weeks' gestation.

ECS, emergency cesarean section; BPD, biparietal diameter.

to be an independent risk factor, as higher BPD values were associated with ECS due to labor arrest. (2) No specific BPD cut-off value was convincingly associated with a 
meaningful increase in the risk of ECS due to labor arrest. These findings are consistent with those of previous studies (Bardin et al. 2016; Karaaslan et al. 2019). Therefore, despite the differences in maternal physique and race, there may be no significant difference in the relationship between BPD (as measured by pre-labor ultrasonography) and the passage of the fetal head through the pelvis. It seems natural to speculate that a large BPD, which may reflect a large fetal head, influences the progression of labor. However, molding of the fetal head during its passage through the maternal pelvis may explain the lack of benefit from the ultrasound evaluation of BPD prior to labor in this context (Bardin et al. 2016); similar findings were obtained in a previous study (Karaaslan et al. 2019). Molding may be a physiological adaptation for protection against fetal or maternal injury. Bardin et al. (2016) reported that unlike the fixed fetal $\mathrm{HC}$, the BPD is flexible to a certain degree owing to molding. The fetal head undergoes molding to enable the passage of its largest diameter (i.e., the BPD) through the pelvis, which may nullify the importance of the antepartum sonographic BPD assessment. Indeed, the fetal BPD alone was poorly predictive of the need for ECS due to labor arrest.

As described above, the findings of the current study are consistent in part with those of previous studies (Bardin et al. 2016; Karaaslan et al. 2019). However, these previous studies had certain limitations, including a small sample size (Karaaslan et al. 2019) and inadequate distinction between the need for ECS due to labor arrest and ECS due to any indication (Bardin et al. 2016). Compared to these studies, our study analyzed more risk factors for ECS due to labor arrest in a relatively large sample of Japanese women.

A short maternal stature was also significantly associated with ECS due to labor arrest in the present study. Several studies have reported that a shorter height of the mother is an important risk factor for labor arrest (McGuinness and Trivedi 1999; Liselele et al. 2000; Kara et al. 2005; Toh-Adam et al. 2012). Maternal height reflects the maternal pelvic size, which is in turn related to dystocia or difficult births; it has, therefore, been shown to be predictive of labor arrest (Toh-Adam et al. 2012). In the clinical setting, several women, particularly those with short stature who have been reported to have large fetal BPDs, have concerns about the possibility of cesarean section delivery. Although multivariable analysis was not possible due to the small sample size, the ability of BPD to predict ECS due to labor arrest was examined. In the present study, based on ROC analysis for 116 women with a short stature $(<150$ $\mathrm{cm}$, see Table 3), we found that a BPD cut-off point of 92 $\mathrm{mm}$ would allow for the maximum number of women to be correctly classified according requiring ECS due to labor arrest. A cut-off value of $92 \mathrm{~mm}$ provided a sensitivity of $66.7 \%$, specificity of $59.1 \%$, positive predictive value of $8.2 \%$, and negative predictive value of $97.0 \%$ (area under the curve: 0.65) (data not shown). Therefore, even though our study population was limited to women with short stature and normal physique, the fetal BPD alone demonstrated poor predictive ability for determining the need for ECS. Obstetricians may find this information useful during the prenatal counseling of women with a short stature.

This study has certain limitations. Firstly, this was a single-center study, and it may be difficult to generalize our results to the general population. A large-scale multicenter, prospective cohort study is required to confirm our results in the general population. Secondly, the definition of labor arrest differs between Japan and other countries (American College of Obstetricians and Gynecologists et al. 2014). In view of the ambiguity in the definition of labor arrest, the difference in the definition of labor arrest between studies needs to be considered. Thirdly, we did not evaluate certain potential risk factors for labor arrest, including premature rupture of membranes (Feinstein et al. 2002; Lee et al. 2012), hydramnios (Feinstein et al. 2002), and labor induction (Feinstein et al. 2002). Therefore, unmeasured confounders may have been associated with labor arrest in this study.

In conclusion, although there is a significant relationship between fetal BPD and the risk of ECS due to labor arrest, the ultrasound assessment does not significantly improve the predictive ability of fetal BPD. In addition, the fetal BPD alone demonstrates poor predictive ability for ECS due to labor arrest. Thus, mothers should be encouraged for attempting vaginal deliveries, even in the case of having babies with large fetal BPD.

\section{Acknowledgments}

We would like to thank the study subjects for allowing the use of their personal data. We would also like to thank Editage (https://www.editage.com) for English language editing.

\section{Conflict of Interest}

The authors declare no conflict of interest.

\section{References}

American College of Obstetricians and Gynecologists; Society for Maternal-Fetal Medicine; Caughey, A.B., Cahill, A.G., Guise, J.M. \& Rouse, D.J. (2014) Safe prevention of the primary cesarean delivery. Am. J. Obstet. Gynecol., 210, 179-193.

Bardin, R., Aviram, A., Meizner, I., Ashwal, E., Hiersch, L., Yogev, Y. \& Hadar, E. (2016) Association of fetal biparietal diameter with mode of delivery and perinatal outcome. Ultrasound Obstet. Gynecol., 47, 217-223.

Boulet, S.L., Alexander, G.R., Salihu, H.M. \& Pass, M. (2003) Macrosomic births in the united states: determinants, outcomes, and proposed grades of risk. Am. J. Obstet. Gynecol., 188, 1372-1378.

Deurenberg, P., Deurenberg-Yap, M. \& Guricci, S. (2002) Asians are different from Caucasians and from each other in their body mass index/body fat per cent relationship. Obes. Rev., 3, 141-146.

Feinstein, U., Sheiner, E., Levy, A., Hallak, M. \& Mazor, M. (2002) Risk factors for arrest of descent during the second stage of labor. Int. J. Gynaecol. Obstet., 77, 7-14. 
Galan, H.L., Pandipati, S. \& Filly, R.A. (2008) Ultrasound Evaluation of Fetal Biometry and Normal and Abnormal Fetal Growth. In Ultrasonography in Obstetrics and Gynecology, 5th ed., Saunders, Philadelphia, PA, pp. 225-265.

Gifford, D.S., Morton, S.C., Fiske, M., Keesey, J., Keeler, E. \& Kahn, K.L. (2000) Lack of progress in labor as a reason for cesarean. Obstet. Gynecol., 95, 589-595.

Kara, F., Yesildaglar, N. \& Uygur, D. (2005) Maternal height as a risk factor for Caesarean section. Arch. Gynecol. Obstet., 271, 336-337.

Karaaslan, O., Islamova, G., Soylemez, F. \& Kalafat, E. (2019) Ultrasound in labor admission to predict need for emergency cesarean section: a prospective, blinded cohort study. J. Matern. Fetal Neonatal Med., doi: 10.1080/14767058.2019. 1687682. [Epub ahead of print].

Kominiarek, M.A., Zhang, J., Vanveldhuisen, P., Troendle, J., Beaver, J. \& Hibbard, J.U. (2011) Contemporary labor patterns: the impact of maternal body mass index. Am. J. Obstet. Gynecol., 205, 244. e1-8.

Lee, S.M., Park, J.W., Park, C.W. \& Yoon, B.H. (2012) "Early rupture of membranes" during induced labor as a risk factor for cesarean delivery in term nulliparas. PLoS One, 7, e39883.

Lipschuetz, M., Cohen, S.M., Israel, A., Baron, J., Porat, S., Valsky, D.V., Yagel, O., Amsalem, H., Kabiri, D., Gilboa, Y., Sivan, E., Unger, R., Schiff, E., Hershkovitz, R. \& Yagel, S. (2018) Sonographic large fetal head circumference and risk of cesarean delivery. Am. J. Obstet. Gynecol., 218, 339. e1-7.

Liselele, H.B., Boulvain, M., Tshibangu, K.C. \& Meuris, S. (2000) Maternal height and external pelvimetry to predict cephalopelvic disproportion in nulliparous African women: a cohort study. BJOG, 107, 947-952.

McGuinness, B.J. \& Trivedi, A.N. (1999) Maternal height as a risk factor for Caesarean section due to failure to progress in labour. Aust. NZ J. Obstet. Gynaecol., 39, 152-154.

Minakami, H., Maeda, T., Fujii, T., Hamada, H., Iitsuka, Y., Itakura, A., Itoh, H., Iwashita, M., Kanagawa, T., Kanai, M., Kasuga, Y., Kawabata, M., Kobayashi, K., Kotani, T., Kudo, Y., et al. (2014) Guidelines for obstetrical practice in Japan: Japan Society of Obstetrics and Gynecology (JSOG) and Japan Association of Obstetricians and Gynecologists (JAOG) 2014 edition. J. Obstet. Gynaecol. Res., 40, 1469-1499.

Nishimura, K., Yoshimura, K., Kubo, T. \& Hachisuga, T. (2016) Objective diagnosis of arrested labor on transperineal ultrasound. J. Obstet. Gynaecol. Res., 42, 803-809.

Ohkuchi, A., Hirashima, C., Takahashi, K., Suzuki, H. \& Matsubara, S. (2017) Prediction and prevention of hypertensive disorders of pregnancy. Hypertens. Res., 40, 5-14.

Perkins, N.J. \& Schisterman, E.F. (2006) The inconsistency of "optimal" cutpoints obtained using two criteria based on the receiver operating characteristic curve. Am. J. Epidemiol., 163, 670-675.

Toh-Adam, R., Srisupundit, K. \& Tongsong, T. (2012) Short stature as an independent risk factor for cephalopelvic disproportion in a country of relatively small-sized mothers. Arch. Gynecol. Obstet., 285, 1513-1516.

Yang, X.J. \& Sun, S.S. (2017) Comparison of maternal and fetal complications in elective and emergency cesarean section: a systematic review and meta-analysis. Arch. Gynecol. Obstet., 296, 503-512. 